\title{
Accuracy of an Experimental Accelerometer for Assessing Countermovement Vertical Jump Height
}

\section{Dimitrije Cabarkapa, Andrew C. Fry, and Matthew J. Hermes}

\begin{abstract}
While force plate technology is the gold standard for assessment of many aspects of vertical jump performance, its cost is prohibitive to a broad spectrum of the population. Accelerometry may be more practical, inexpensive, and provide a simple solution that allows hands-on practitioners to readily assess vertical jump performance acutely and over time. Thus, the purpose of this study was to examine the accuracy of an experimental accelerometer for testing vertical jump heights derived from flight times when compared to a laboratory-based force plate system as a criterion measure. Fifteen subjects performed three sets of three nonconsecutive maximal countermovement vertical jumps while standing on the force plate. The accelerometer device sampling at $100 \mathrm{~Hz}$ was placed on the anterior abdomen immediately inferior to the umbilicus and secured with an elastic band. Both devices recorded the data simultaneously. The experimental accelerometer was an appropriate tool for the assessment of vertical jump height; however, it significantly overestimated actual vertical jump heights by an average of $3.1 \mathrm{~cm}$. This consistent discrepancy in the measurement may be easily fixed by a simple algorithm correction and should not present an issue in the practical setting where ease of use and the ability to provide immediate feedback regarding an athlete's performance is of critical importance.
\end{abstract}

Keywords: sport, testing, performance, technology, sport science

\footnotetext{
Dimitrije Cabarkapa, MS, CSCS, NSCA-CPT, USAW, is a doctoral candidate and graduate teaching assistant in the Jayhawk Athletic Performance Laboratory, Department of Health, Sport, and Exercise Sciences at the University of Kansas. His research interests are primarily focused on the kinetic and kinematic analysis of various sport-specific motions, performance tracking technologies, and physiological responses to exercise. Email: dcabarkapa@ku.edu

Andrew C. Fry, PhD, CSCS*D, FNSCA, is a professor in the Department of Health, Sport, and Exercise Science and the director of the Jayhawk Athletic Performance Laboratory at the University of Kansas. His research interests are primarily focused on resistance exercise, skeletal muscle physiology, endocrine responses to exercise, and overtraining. Email: acfry@ku.edu

Matthew J. Hermes, MS, USAW, is a doctoral student and graduate teaching assistant in the Jayhawk Athletic Performance Laboratory, Department of Health, Sport, and Exercise Sciences at the University of Kansas. His research interests are primarily focused on weightlifting, exercise training modalities, and adaptations to training. Email: m602h699@ku.edu
} 


\section{Introduction}

A variety of methods exist to assess lower body power output in athletes. These include jumping, weightlifting, and cycle ergometry assessments (ÇakirAtabek, 2014; Carlock et al., 2004; Hoffman et al., 2000). Despite differences in testing methodologies, previous research has indicated performances on these assessments were highly correlated with each other. Carlock et al. (2004) explored the relationship between vertical jump, estimated vertical jump power, and weightlifting performance. The authors found strong correlations between the estimated peak power from both static and countermovement jumps and weightlifting performance $(r \geq 0.90)$. Additionally, the vertical jump has been shown to be positively correlated with both mean and peak power outputs attained during Wingate testing (Çakir-Atabek, 2014; Hoffman et al., 2000). This suggests vertical jump assessments are a valid field test for evaluating lower body power output in athletes. Furthermore, vertical jump tests may be more feasible in settings where access to specialized equipment or limited space may be constraints for coaches.

Vertical jump performance can be assessed in a variety of ways (Buckthorpe et al., 2012; Menzel et al., 2010; Whitmer et al., 2015). A laboratory force plate has been considered the gold standard for vertical jump performance assessment, as it allows for precise measurement of ground reaction forces and flight times (Menzel et al., 2010). However, this may be impractical in many settings as this method requires access to expensive testing equipment. Other more affordable and user-friendly methods for assessment of jumping performance exist and have been compared to a laboratory force plate system. In a comparison of four devices (belt mat, contact mat, portable force plate, and Vertec) to a laboratory-based force plate, vertical jump performance was highly correlated $(r \geq 0.90)$ to force plate measurements across all devices (Buckthorpe et al., 2012). It is also important to note there are two ways to measure vertical jump height: one using hand reach height (e.g., using a Vertec) and one using height of the center of mass (e.g., using a force plate). While these two methods should be highly correlated, measures using center of mass will be lower. With this in mind, reported flight times $\left(r^{2}=0.995\right)$ and jump heights $\left(r^{2}=0.997\right)$ were highly correlated between a contact mat and a laboratory-based force plate system but were significantly longer by over $100 \mathrm{~ms}$ and higher by $16 \mathrm{~cm}$ for the contact mat (Whitmer et al., 2015). It was speculated that flight times for the contact mat were systematically increased by approximately $100 \mathrm{~ms}$ to produce agreement with Vertec-derived jump reach heights; however, this appeared to underestimate vertical jump heights for high performers (e.g., > $0.70 \mathrm{~m}, 27.6$ in; Whitmer et al., 2015).

While some alternative methods for vertical jump height assessment provide ease of use or lower cost when compared to gold standard technologies, the 
ability to evaluate force-time characteristics may be impaired. To provide an alternative to a force plate that is not solely restricted to laboratory usage and can assess force-time characteristics, accelerometry has been used in a variety of cases (Castagna et al., 2013; Heredia-Jimenez \& Orantes-Gonzales, 2020). Some of the most commonly chosen anatomical locations for wearable accelerometer placement include scapulae, sternum, or waist near the center of mass (Gómez-Carmona et al., 2020). Despite some practical advantages, accelerometers have yielded inconsistent results when compared to a laboratory force plate system. This has been mainly attributed to an inability to measure multisegmented body accelerations (Nedergaard et al., 2017). A triaxial accelerometer consistently overestimated peak force, rate of force development, peak power, flight time, and vertical displacement when compared to a force plate and linear position transducer (Ruben et al., 2011). Additionally, peak concentric forces from an accelerometer were consistently higher when compared to a force plate, despite a good agreement between the devices on minimum eccentric force (Howard et al., 2014). Castagna et al. (2013) conducted a study on an elite cohort of rugby players and found that flight times reported by a wearable accelerometer during maximal countermovement vertical jumps were notably greater when compared to an optical jump height system and a laboratory-based force plate system as a criterion measure. While both optical and accelerometer systems displayed a strong correlation with vertical jump flight times reported by the force plate, the accelerometer system reported an average of $7.2 \%$ greater vertical jump heights values when compared to the optical jump height system (Castagne et al., 2013). In a similar manner, Heredia-Jimenez \& Orantes-Gonzales (2020) examined the accuracy of the wearable accelerometer system technology for vertical jump height assessment based on the flight times within a cohort of recreationally active individuals. The researchers found the accelerometer system overestimated vertical jump heights by approximately $7 \mathrm{~cm}$ when compared to a laboratory-based force plate system (Heredia-Jimenez \& Orantes-Gonzales, 2020). Moreover, agreements in vertical jump height measurements have been noted between accelerometers and various motion capture systems (Grainger et al., 2020; Mahmoud et al., 2015), but it is not known how these devices compare to force plate evaluation.

The exponential growth of technology over the last decade has allowed for the development and advancement of innovative sports performance monitoring methodologies. While the force plate remains the gold standard for assessment of vertical jump performance, its cost is prohibitive to a broad spectrum of the population. Budget constraints and limited capital funding may put a force plate system out of reach for most institutions on various levels of athletic competition. Thus, accelerometry may be a practical, inexpensive, and simple solution 
that allows hands-on practitioners to assess vertical jump performance acutely and over time. The purpose of this study was to examine the accuracy of an innovative experimental accelerometer for testing vertical jump heights derived from flight times when compared to a laboratory-based force plate system as a criterion measure. It was hypothesized that the accelerometer would demonstrate a good criterion validity and yield similar vertical jump height measurements.

\section{Methods}

\section{Participants}

Fifteen healthy recreationally active subjects, ten female $(\overline{\mathrm{x}} \pm \mathrm{SD}$; age $=22.2 \pm 2.9$ years, body mass $=69.9 \pm 7.7 \mathrm{~kg}$, height $=168.1 \pm 7.6 \mathrm{~cm})$ and five male (age $=$ $22.2 \pm 3.3$ years, body mass $=84.8 \pm 19.5 \mathrm{~kg}$, height $=179.8 \pm 5.2 \mathrm{~cm}$ ), volunteered to participate in this study. Before any testing procedures, each subject reviewed and signed the informed consent form. To assure safety of the subjects and to optimize testing conditions, subjects with musculoskeletal injuries were not permitted to participate in this study. All testing procedures were previously approved by the researchers' university's Institutional Review Board.

\section{Protocol}

Upon arrival at the laboratory, participants performed a standardized warmup consisting of a five-minute treadmill run at self-selected moderate velocity and a set of dynamic motions (high knees, butt kicks, forward lunges, lateral skips, A-skips, Spiderman crawl, and tuck jumps). The accelerometer device (Strive, Beta Version 1.0, Bothell, WA, USA) was placed $3 \mathrm{~cm}$ inferior to the umbilicus and secured with an elastic band. To fit a diverse group of participants, the bands were offered in different sizes (small, medium, large, extra-large). The raw accelerometer data was sampled at $100 \mathrm{~Hz}$. A uni-axial force plate (Rice Lake Weighing Systems, Rice Lake, WI, USA) and data acquisition system (BioPac MP 150 System, Goleta, CA, USA) sampling at 1,000 Hz was used to measure flight times from ground reaction forces. While standing on the force plate, each participant completed three sets of three non-sequential maximal countermovement vertical jumps. Each set was separated by a 1-2 minute recovery period to minimize fatigue. Both devices collected the data simultaneously. The overall number of jumps participants performed was 135 . After data collection, the force plate data was converted to $100 \mathrm{~Hz}$ to match the sampling rate of the accelerometer. Flight times were obtained from both devices and maximal vertical jump heights were calculated from the following equation. 


$$
\begin{gathered}
H=H_{0}+V_{0} \cdot t+1 / 2 \cdot a \cdot t^{2} \\
\text { H: jump height, } H_{0} \text { : take-off height, } V_{0}: \text { take-off velocity, } a: 9.81 \mathrm{~m} / \mathrm{s}^{2} \text {, t: flight time duration }
\end{gathered}
$$

\section{Statistical Analysis}

All descriptive data is reported as $\overline{\mathrm{x}} \pm \mathrm{SD}$. Independent t-tests were used to determine statistically significant differences in derived vertical jump heights for the force plate and the accelerometer. Pearson product-moment correlation coefficient for linear regression was used to determine the degree of association and explained variance between the two data sets. The standard error of the estimate (SEE) was calculated as a measure of the accuracy of the calculations, and Bland-Altman plots depicted the agreement of measurements from the devices. Additionally, to further determine agreement and test for internal consistency and accuracy of the measurements, intra-class correlation coefficients (ICC) and Cronbach alpha values were calculated. Cohen's D effect sizes were calculated to compare difference between the means. Statistical significance was set priori to $p<0.05$. Based on prior work in our laboratory (Whitmer et al., 2015), the required sample size needed to maintain 95\% confidence was 17 subjects. As such, our sample was similar. All statistical analyses were completed with Microsoft Excel 2016 (Microsoft Corporation, Redmond, WA, USA) and SPSS Version 25.0 statistical software package (SPSS Inc., Chicago, IL, USA).

\section{Results}

Due to three highly prominent outlier data points, one male participant was excluded from the final data analysis. Additionally, six jump trials were not recorded due to researcher error in delayed accelerometer activation, leaving 120 jump trials for the final data analysis. The average countermovement vertical jump heights and flight times computed from the force plate and the experimental accelerometer are presented in Table 1. Independent t-tests indicated significant differences in countermovement vertical jump heights and flight times between the two methods of assessment $(p<0.001)$. The scatter plot of the linear regression for vertical jump heights is presented in Figure 1. When compared to the force plate as the criterion measurement, the accelerometer overestimated vertical jump heights by an average of $0.031 \mathrm{~m}(3.1 \mathrm{~cm})$, which corresponds to approximately $0.024 \mathrm{sec}(24 \mathrm{~ms})$ of the flight time. However, the standard error of estimate magnitude was only $0.026 \mathrm{~m}(2.6 \mathrm{~cm})$, indicating a small prediction error. Pearson correlation coefficient $(r=0.927)$ and explained variance $\left(r^{2}=0.859\right)$ magnitudes indicated strong association and substantial goodnessof-fit of the linear regression model. Additionally, the ICC coefficient indicated 
strong agreement $(\mathrm{ICC}=0.913)$ between the measurements and Cronbach's alpha value $(\alpha=0.962)$ denoted excellent internal consistency. A Bland-Altman plot showing the agreement between the devices is presented in Figure 2.

Table 1. Vertical jump flight times (sec) and vertical jump heights $(\mathrm{m})$ for both devices examined in this study $(\overline{\mathrm{x}} \pm$ SD $[95 \% \mathrm{Cl}])$.

\begin{tabular}{lccc} 
& Accelerometer & Force Plate & Effect Size \\
\hline Flight Time (sec) & $0.537 \pm 0.049^{*}$ & $0.513 \pm 0.054$ & 0.465 \\
& {$[0.511-0.563]$} & {$[0.485-0.541]$} & \\
Vertical Jump Height (m) & $0.357 \pm 0.066^{*}$ & $0.326 \pm 0.069$ & 0.459 \\
& {$[0.322-0.392]$} & {$[0.290-0.362]$} & \\
\hline
\end{tabular}

${ }^{*}$ significant difference $(p<0.05)$

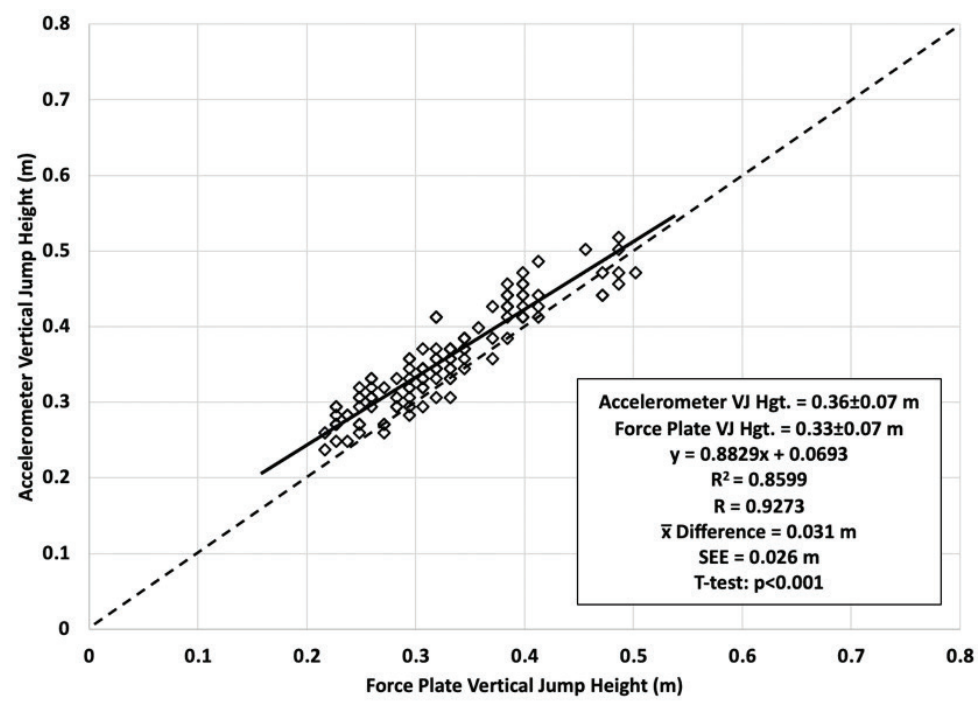

Figure 1. Comparison of vertical jump heights determined from the force plate and the experimental accelerometer.

Dashed line $=$ line of agreement. Solid bold line $=$ regression line . 


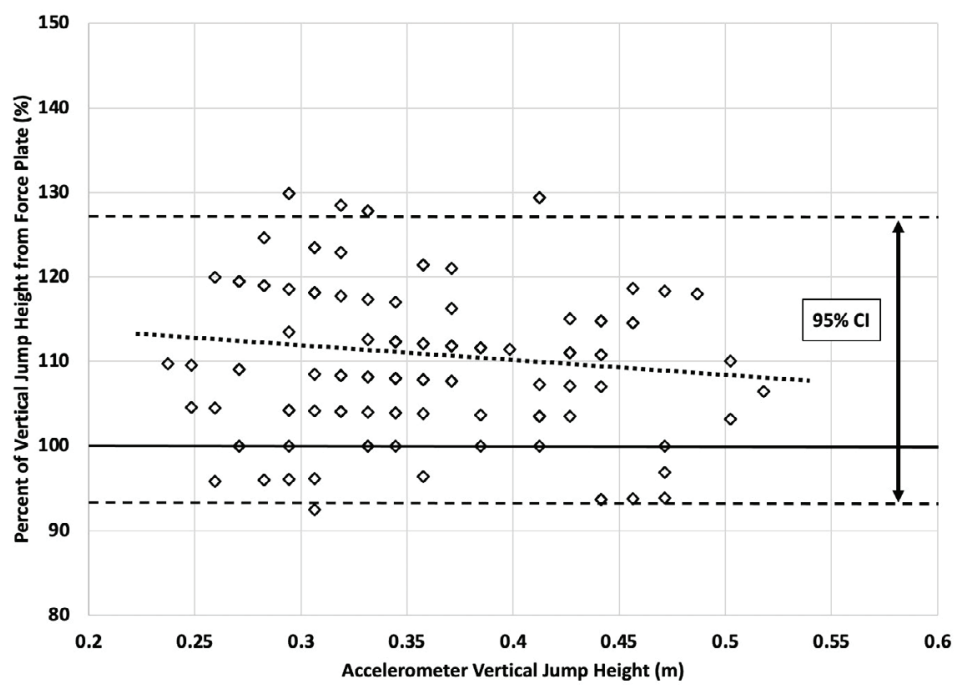

Figure 2. Bland-Altman plot demonstrating agreement in vertical jump heights measurements between the force plate and the experimental accelerometer.

Dashed lines $=95 \% \mathrm{Cl}$. Dotted line $=$ regression line. Solid bold line $=$ line of agreement .

\section{Discussion}

Unlike initially hypothesized, the findings of this study identified a statistically significant difference in vertical jump height measurements estimated by the experimental accelerometer compared to a laboratory-based force plate system as the criterion measure. The experimental accelerometer overestimated vertical jump heights by an average of $3.1 \mathrm{~cm}$. The observed trend of overestimation in vertical jump height measurements seems to be in the agreement with previous studies (Castagna et al., 2013; Heredia-Jimenez \& Orantes-Gonzales, 2020; Monnet et al., 2014; Ruben et al., 2011). Nevertheless, the magnitude of the overestimation for the specific accelerometer device examined in the present study is approximately two-fold smaller than reported in the previously mentioned studies, indicating improved vertical jump height measurement accuracy (Heredia-Jimenez \& Orantes-Gonzales, 2020; Monnet et al., 2014).

Based on the findings from the current study, validity of the experimental accelerometer used for vertical jump height assessment seems to be questionable from the statistical significance point of view. However, being solely focused on the statistical significance may not be the only appropriate way to interpret the scientific findings. Sport scientists and practitioners need to be aware of 
the practical significance and practical application of our findings, which occasionally may be of greater relevance, especially in the sport and strength and conditioning setting. A laboratory-based force plate system is an expensive piece of equipment. Despite being considered a gold standard measurement for vertical jump height assessment, it requires the expertise of a trained practitioner or sport scientist to collect and analyze the data. Due to these constraints, the user-friendliness of force plate technology is sometimes reduced. In order to track athletes' progress and obtain insight on their lower body power production, coaches need instantaneous feedback. Therefore, statistically significant differences regarding the validity of the experimental accelerometer for vertical jump height assessment may not present a large issue in a practical setting and may still provide coaches and athletes with useful information necessary for long-term performance enhancement.

Considering the extremely strong correlations between the flight times and vertical jump heights from the experimental accelerometer and the force plate, this issue might be easily resolved by a simple correction in the mathematical algorithm. When using the experimental accelerometer, it might be appropriate to subtract the mean difference in flight times $(24 \mathrm{~ms})$ or jump heights $(3.1 \mathrm{~cm})$ found between the two devices in order to obtain more accurate vertical jump height measurements. However, this adjustment may be too simplistic. Whitmer et al. (2015) suggested a similar approach to correct for systematic error in vertical jump height estimations assessed with a contact jump mat. The authors found vertical jump performance was underestimated to a greater extent with increasing jump heights, up to approximately $15.2 \mathrm{~cm}(6.0 \mathrm{in})$ in measurement error at vertical jump heights of $1.1 \mathrm{~m}$ (43.3 in) (Whitmer et al., 2015). Based on the findings of the present study, vertical jump height values for higher performers may be underestimated in a similar manner. The regression lines in Figures 1 and 2 suggest this may be the case. Thus, additional testing is needed to determine if the observed difference between the experimental accelerometer and a laboratory-based force plate system remains constant for subjects with a greater athletic ability since the conclusions in this study were made on a cohort of recreationally active individuals. It is likely that any correction factors would need to be relative to the measured vertical jump height and would require a regression-derived correction factor.

Besides the algorithms used to estimate vertical jump heights based on flight times, the anatomical location of the accelerometer may further influence the validity of the measurements. Body-worn accelerometers measure the acceleration from the specific body segment and might not be an adequate tool for assessing the whole body's multisegmented motion (Nedergaard et al., 2017). In a recently published systematic review, Gómez-Carmona et al. (2020) indicated some 
of the most commonly chosen locations for accelerometer placement include scapulae, sternum, or waist near the center of mass. The specified location for placement of the experimental accelerometer examined in this study was the anterior waist, where the device was secured with an adjustable elastic band. Anatomical locations with a lower tendency for an accumulation of adipose tissue are commonly chosen to avoid unnecessary accelerometer movement that can lead to an increase in the error of measurement. The authors believe this may be a contributing factor for several of the outlying measurements observed in the present study.

Additionally, while securing the accelerometer with an elastic band is very practical, attaching the accelerometer more securely may further improve the measurement accuracy. Possible locations could include embedding the accelerometer in personally fitted compression shorts at the waist or choosing different anatomical spots for its placement, such as at the greater trochanter or on the back (near L3-L5). Preliminary findings suggest the kinetic and kinematic data from a vertical jump are highly dependent on the anatomical location monitored, the specific variable measured, and the technology used for assessment (unpublished data). Further research needs to examine if these suggestions can diminish systematic error and improve the accuracy of accelerometry technology for vertical jump height assessment based on the flight time.

To our knowledge, this was the first study that examined the accuracy of the experimental accelerometer for assessment of vertical jump height when compared to a laboratory-based force plate system as a criterion measure. However, certain limitations are present. The sample size of subjects recruited could have been slightly larger. It should have included a larger number of participants from more diverse age-range groups. Additionally, this investigation was solely focused on assessing vertical jump capabilities of recreationally active individuals. Therefore, future research should focus on addressing the previously mentioned limitations and determine if agreement in the measurements remains consistent regardless of a participant's age group and physical activity level (i.e., untrained, recreationally active, athlete).

Based on the findings of the present study, the authors conclude the experimental accelerometer demonstrated as an acceptable tool for assessment of vertical jump height. However, due to the tendency to overestimate vertical jump heights on average by $3.1 \mathrm{~cm}$, the validity of the device was slightly impaired. While this discrepancy in measurement may be easily fixed by a simple algorithm correction, it may not be an issue in a practical setting where user-friendliness and the ability to provide instantaneous feedback regarding an athlete's performance is of critical importance. 


\section{Practical Application}

While force plate technology has been considered the gold standard for the assessment of many aspects of vertical jump performance, a need for accurate, practical, and inexpensive technology remains a challenge. The findings of this study indicate that the experimental accelerometer demonstrated as an adequate method for vertical jump performance assessment. Due to lower cost and enhanced user-friendliness, this device can be an alternative solution for institutions where financial constraints and budget limitations might be an issue. By being granted access to this type of accelerometry technology, coaches and hands-on practitioners can readily assess vertical jump performance and assure optimal long-term athlete development.

\section{References}

Buckthorpe, M., Morris, J., \& Folland, J. P. (2012). Validity of vertical jump measurement devices. Journal of Sports Sciences, 30(1), 63-69. https://doi.org/10.1080/02640414.2011.624539

Carlock, J. M., Smith, S. L., Hartman, H. J., Morris, R. T., Ciroslan, D. A., Pierce, K. C., Newton, R. U., Harman, E. A., Sands, W. A., \& Stone, M. H. (2004). The relationship between vertical jump power estimates and weightlifting ability: A field-test approach. Journal of Strength and Conditioning Research, 18(3), 534-539. https://doi.org/10.1519/R-13213.1

Castagna, C., Ganzetti, M., Ditroilo, M., Giovannelli, M., Rocchetti, A., \& Manzi, V. (2013). Concurrent validity of vertical jump performance assessment systems. Journal of Strength and Conditioning Research, 27(3), 761-768. https://doi.org/10.1519/JSC.0b013e31825dbcc5

Çakir-Atabek, H. (2014). Relationship between anaerobic power, vertical jump and aerobic performance in adolescent track and field athletes. Journal of Physical Education and Sport, 14(4), 643-648. https://doi.org/10.7752/jpes.2014.04100

Gómez-Carmona, C. D., Bastida-Castillo, A., Ibáñez, S. J., \& Pino-Ortega, J. (2020). Accelerometry as a method for external workload monitoring in invasion team sports. A systematic review. PloS one, 15(8), e0236643. https://doi.org/10.1371/journal.pone.0236643

Grainger, M., Weisberg, A., Stergiou, P., \& Katz, L. (2020). Comparison of two methods in the estimation of vertical jump height. Journal of Human Sport and Exercise, 15(3), 623-632. https:// doi.org/10.14198/jhse.2020.153.12

Heredia-Jimenez, J., \& Orantes-Gonzalez, E. (2020). Comparison of three different measurement systems to assess the vertical jump height. Revista Brasileira de Medicina do Esporte, 26(2), 143-146. http://dx.doi.org/10.1590/1517-869220202602185305

Hoffman, J. R., Epstein, S., Einbinder, M., \& Weinstein, Y. (2000). A comparison between the Wingate anaerobic power test to both vertical jump and line drill tests in basketball players. Journal of Strength and Conditioning Research, 14(3), 261-264.

Howard, R., Conway, R., \& Harrison, A. J. (2014). Estimation of force during vertical jumps using body fixed accelerometers. 25th IET Irish Signals \& Systems Conference 2014 and 2014 China-Ireland International Conference on Information and Communications Technologies (ISSC 2014/CIICT 2014), Limerick, 102-107. https://doi.org/10.1049/cp.2014.0667

Mahmoud, I., Othman, A. A. A., Abdelrasoul, E., Stergiou, P., \& Katz, L. (2015). The reliability of a real time wearable sensing device to measure vertical jump. Procedia Engeniring, 112(1), 467-472. https://doi.org/10.1016/j.proeng.2015.07.226 
Menzel, H.-J., Chagas, M. H., Szmuchrowski, L. A., Araujo, S. R., Campos, C. E., \& Giannetti, M. R. (2010). Usefulness of the jump-and-reach test in assessment of vertical jump performance. Perceptual and Motor Skills, 110(1), 150-158. https://doi.org/10.2466/pms.110.1.150-158

Monnet, T., Decatoire, A., \& Lacouture, P. (2014). Comparison of algorithms to determine jump height and flight time from body mounted accelerometers. Sports Engineering, 17(4), 249-259. https://doi.org/10.1007/s12283-014-0155-1

Nedergaard, N. J., Robinson, M. A., Eusterwiemann, E., Drust, B., Lisboa, P. J., \& Vanrenterghem, J. (2017). The relationship between whole-body external loading and body-worn accelerometry during team-sport movements. International Journal of Sports Physiology and Performance, 12(1), 18-26. https://doi.org/10.1123/ijspp.2015-0712

Ruben, R. M., Saffel, H., McCrory, J. L., Cormie, P., \& Haff, G. G. (2011). Comparison of accelerometer based vertical jump assessments to a linear position transducer plus force plate system. Journal of Strength and Conditioning Research, 25, S37. https://doi.org/10.1097/01. JSC.0000395636.70853.92

Whitmer, T. D., Fry, A. C., Forsythe, C. M., Andre, M. J., Lane, M. T., Hudy, A., \& Honnold, D. E. (2015). Accuracy of a vertical jump contact mat for determining jump height and flight time. Journal of Strength and Conditioning Research, 29(4), 877-881. https://doi.org/10.1519/ JSC.0000000000000542

\section{Acknowledgments}

The authors would like to thank all individuals who volunteered to participate in this study as well as Nikola Mrvaljevic, Zach Shelly, and Quinn AbrahamsVaughn for assistance with the data collection process. 